CERN-TH/95-44

\title{
Non-Unified Sparticle and Particle Masses in Unified Theories
}

\author{
Savas Dimopoulos 円 and Alex Pomarol \\ Theory Division, CERN \\ CH-1211 Geneva 23, Switzerland
}

\begin{abstract}
We give examples of minimal extensions of the simplest SU(5) SUSYGUT in which all squarks and sleptons of a family have different tree level masses at the unification scale. This phenomenon is general; it occurs when the quarks and leptons are the light remnants of a theory which contains extra heavy families at the unification scale. The examples have interesting relations between Yukawa couplings: In one model the ratio of the top to bottom Yukawas is as large as $\simeq 3$, partly accounting for the large $m_{t} / m_{b}$. Another gives $m_{b} / m_{\tau}$ between $2 / 3$ and 1 ; this relaxes the strict bounds on the top mass and neutrino properties that come from $b-\tau$ unification. Still another allows $m_{s} / m_{\mu}$ to be between $1 / 6$ and 1 and evades the potentially problematic GUT relation of $m_{s}=m_{\mu}$. The final example has horizontal sparticle splittings in spite of the existence of horizontal symmetries.
\end{abstract}

CERN-TH/95-44

February 1995

\footnotetext{
${ }^{1}$ On leave of absence from the Physics Department, Stanford University, Stanford CA 94305, USA.
} 


\section{Sparticle masses as a probe of unification}

It is unlikely that we will ever build a microscope that allows us to directly study physics at Planckean distances $\sim 10^{-33} \mathrm{~cm}$. The best we hope for is to directly look at physics near the $\mathrm{TeV}$ scale and then use theoretical ideas, such as the desert hypothesis, to extrapolate further. The desert hypothesis acts as a microscope which magnifies by 14 orders of magnitude without running into any obstacles and allows us to look at Planckean distances. Conversely, the desert hypothesis allows theoretical ideas near the Planck mass to be translated into low-energy predictions that can be directly compared to experiment. This speculative program has had a remarkable success: it predicted a value for the weak mixing angle in supersymmetric unified theories (SUSY-GUTs) [1, 2, [3] which was subsequently confirmed by experiment [4]. This gave a vote of confidence to the desert hypothesis, the existence of light sparticles and the idea of gauge coupling unification. Of course, there are many more parameters in addition to gauge couplings: fermion and sparticle masses and mixing angles add up to a grand total of 110 physical parameters just in the supersymmetric flavor sector. According to the desert hypothesis, each of these carries direct information about the structure of the theory at Planckean distances.

One objective of this paper is to present some counterexamples to simple expectations for the sparticle masses in large classes of unified theories. The earliest hypothesis on sparticle masses is called universality; it was motivated from the need to suppress flavor violating processes [1] and postulates that all squarks and sleptons are degenerate at the unification scale. Although this strong form of universality is neither necessary nor likely, it is widely believed that a more restricted form is always valid in unified theories: sparticles belonging to the same SU(5) multiplet are degenerate at the unification scale. This is considered a direct consequence of unification which will be experimentally checked if and when sparticles are discovered at LHC and NLC and their masses are known with some precision. Furthermore, since the 15 sparticle species of 3 families fall into $6 \mathrm{SU}(5)$-multiplets we should have 9 non-trivial relations that test unification by looking at sparticle masses, according to this belief.

We demonstrate that, because the GUT group is spontaneously broken, there is no good reason for this belief. Sparticles, such as $\tilde{b}_{R}$ and $\tilde{\tau}_{L}$, which 
by virtue of their gauge and family quantum numbers can be grouped into an irreducible $\mathrm{SU}(5)$ multiplet do not necessarily originate from one such multiplet; they could come about from a linear combination of several identical multiplets. This can produce non-degeneracy among sparticles of the same generation and complementary SU(5) quantum numbers 1 . The examples that we present are not just of mathematical interest; the sparticle splittings occur precisely in theories (and for the same reasons) that produce interesting and desirable relations among fermion masses.

An essential ingredient for these effects is the non-universality of sparticle masses belonging to different $\mathrm{SU}(5)$ multiplets. This is so generic that it hardly needs to be justified. Sparticles in different multiplets of the unified group have no symmetry reason to be degenerate at the unification scale or any other scale; even if they are assumed to be degenerate at the Planck scale their different interactions will split them by the time they reach the unification mass [5, 6]. These splittings are typically large due to the large size of the representations in unified groups [6] Intermultiplet sparticle splittings can induce intramultiplet splittings in higher rank gauge groups such as $\mathrm{SO}(10), \mathrm{E}(6)$ and beyond [8]; this mechanism, in contrast to the one presented here, is not operative in $\mathrm{SU}(5)$.

\section{A model with $2 / 3<\mathbf{m}_{\mathrm{b}} / \mathbf{m}_{\tau}<1$}

The model is a minimal extension of the SU(5) SUSY-GUT [1]. Consider a $\mathrm{SU}(5)$ theory with just the third generation 1 consisting of a $\overline{\mathbf{5}}_{1}$ and $\mathbf{1 0}_{1}$, the usual Higgs fiveplet $\mathbf{H}$ and antifiveplet $\overline{\mathbf{H}}$, and the adjoint $\mathbf{2 4}$ that breaks $\mathrm{SU}(5)$ down to $\mathrm{SU}(3) \times \mathrm{SU}(2) \times \mathrm{U}(1)$ at the unification scale $M_{G}$ by acquiring a vacuum expectation value $(\mathrm{VEV})$ that points in the hypercharge direction:

$$
\langle\mathbf{2 4}\rangle=V_{24} \mathbf{Y} \equiv V_{24} \operatorname{diag}(2,2,2,-3,-3) .
$$

The bottom and tau masses are given by the superpotential

$$
W=h \mathbf{1 0}_{1} \overline{\mathbf{H}} \overline{\mathbf{5}}_{1},
$$

\footnotetext{
${ }^{2}$ These are sparticles whose combined quantum numbers complete an irreducible SU(5) multiplet.

${ }^{3}$ Such splittings also occur in string theories [7].

${ }^{4}$ Adding the two light generations will not change the conclusions.
} 
and are equal at the unification scale [9]. Now add an extra fiveplet and antifiveplet denoted by $\boldsymbol{5}_{H}$ and $\overline{\boldsymbol{5}}_{2}$ with the following couplings:

$$
W=\mathbf{5}_{H}\left[M \overline{\mathbf{5}}_{1}+\lambda \mathbf{2 4} \overline{\mathbf{5}}_{2}\right]+h \mathbf{1 0} \overline{\mathbf{H}}_{1} \overline{\mathbf{5}}_{1}
$$

where $M$ is near the unification mass. One linear combination of $\overline{\mathbf{5}}_{1}$ and $\overline{\mathbf{5}}_{2}$ will acquire a large mass of order $\sim M_{G}$. The orthogonal combination will be part of the low-energy spectrum. It contains the right-handed bottom quark and the tau lepton doublet which are denoted by $D^{c}$ and $L$ respectively; because the hypercharges of $D^{c}$ and $L$ differ, it follows from eqs. (11) and (3) that they will be different linear combinations of the corresponding states in $\overline{\mathbf{5}}_{1}$ and $\overline{\mathbf{5}}_{2}$ :

$$
\left(\begin{array}{c}
D^{c} \\
L
\end{array}\right)=-\sin \theta_{Y} \overline{\mathbf{5}}_{1}+\cos \theta_{Y} \overline{\mathbf{5}}_{2}
$$

where

$$
\sin \theta_{Y}=\frac{\rho \mathbf{Y}}{\sqrt{1+\rho^{2} \mathbf{Y}^{2}}}
$$

with $\rho=\lambda V_{24} / M$.

Since $\overline{\mathbf{5}}_{1}$ and $\overline{\mathbf{5}}_{2}$ are in different representations of $\mathrm{SU}(5)$, they have, in general, different soft SUSY breaking masses at $M_{G}$ ๆ:

$$
\mathcal{L}_{\text {soft }}=m_{1}^{2}\left|\overline{\mathbf{5}}_{1}\right|^{2}+m_{2}^{2}\left|\overline{\mathbf{5}}_{2}\right|^{2}
$$

Since the light combination is given by (4), one has

$$
\begin{aligned}
& m_{\tilde{b}_{R}}^{2}=m_{2}^{2}+s_{b_{R}}^{2}\left(m_{1}^{2}-m_{2}^{2}\right), \\
& m_{\tilde{\tau}_{L}}^{2}=m_{2}^{2}+s_{\tau_{L}}^{2}\left(m_{1}^{2}-m_{2}^{2}\right),
\end{aligned}
$$

where $b_{R} \in D^{c}, \tau_{L} \in L$ and $s_{a}$ is given by

$$
s_{a}=\sin \theta_{Y_{a}}=\frac{\rho Y_{a}}{\sqrt{1+\rho^{2} Y_{a}^{2}}},
$$

and $Y_{a}$ is the hypercharge of $a$. Therefore, the squark and slepton masses differ at $M_{G}$; their fractional mass-splitting, for $\Delta>0$, is given by

$$
\frac{m_{\tilde{\tau}_{L}}^{2}-m_{\tilde{b}_{R}}^{2}}{m_{\tilde{\tau}_{L}}^{2}}=\frac{s_{\tau_{L}}^{2}-s_{b_{R}}^{2}}{\Delta+s_{\tau_{L}}^{2}},
$$

\footnotetext{
${ }^{5}$ Even if they were equal near the Planck scale, renormalization effects can induce large splittings at $M_{G}$ 河, 6.
} 
where $\Delta=m_{2}^{2} /\left(m_{1}^{2}-m_{2}^{2}\right)$. Eq. (9) is plotted in Fig. 1 as a function of $\rho$ and for different values of $\Delta$. It increases for small values of $\Delta$. For $\Delta \sim 0.2$ and $\rho \sim 0.3$, the fractional mass-splitting is $\sim 30 \%$.

The fermion masses arise from the Yukawa coupling $\mathbf{1 0}_{1} \overline{\mathbf{H}} \overline{\mathbf{5}}_{1}$ :

$$
\begin{aligned}
& m_{b}=h s_{b_{R}}\langle\overline{\mathbf{H}}\rangle, \\
& m_{\tau}=h s_{\tau_{L}}\langle\overline{\mathbf{H}}\rangle,
\end{aligned}
$$

that leads to the ratio between the bottom and tau mass

$$
\frac{m_{b}}{m_{\tau}}=\frac{s_{b_{R}}}{s_{\tau_{L}}}=\frac{2}{3} \sqrt{\frac{1+9 \rho^{2}}{1+4 \rho^{2}}} .
$$

This ratio tends to $2 / 3$ and 1 in the small and large $\rho$ limit respectively. From eqs. (9) and (11), one can see that the scalar mass-splitting is correlated to the fermion mass-splitting. This is shown in Fig. 1 where the dashed line represents the ratio $m_{b} / m_{\tau}$. The maximum values for the scalar mass-splitting correspond to $m_{b} / m_{\tau} \sim 0.7-0.8$.

Thus this model, although it is a minimal perturbation of the SU(5) SUSYGUT [1], easily accommodates values of $m_{b} / m_{\tau}$ that are between $2 / 3$ and 1 . This has at least two interesting implications:

- It relaxes the strong constraints on the top mass that arise from bottomtau unification [10] and thus reduces the degree of fine-tuning that is usually required in these models to achieve electroweak symmetry breaking (because the $\mu$ parameter is typically larger than $m_{Z}$ [11]).

- It relaxes the constraints on the neutrino properties that arise from bottomtau unification for $\tan \beta<10$ [12].

It is now easy to see how the sparticle and particle splittings came about in this model. Although the right-handed bottom and the tau lepton doublet - by virtue of their family and gauge quantum numbers - appear to belong to the same $\overline{\mathbf{5}}$ of SU(5), they in fact, because of their different hypercharges, came from two different linear combinations of a pair of $\overline{\mathbf{5}}$ s. This causes SU(5) and $S U(4)_{P S}$ breaking effects in sparticles and particles to be felt at the tree level, since they occur at the very basic stage of defining the light states of the theory. This mechanism has been used to produce non-trivial fermion mass relations for the two light generations [13, 14], but not for the third family. 


\section{A model with $1 / 6<\mathbf{m}_{\mathrm{s}} / \mathbf{m}_{\mu}<1$}

The main ingredient of the previous section is that the light states $D^{c}$ and $L$ emerge from different linear combinations of states in two different $\overline{\mathbf{5}} \mathrm{s}$. The same idea can be implemented for the states in the decouplets. Add to the previous model an extra $\overline{\mathbf{1 0}}_{H}$ and $\mathbf{1 0}_{2}$ and, instead of eq. (3), consider the superpotential:

$$
W=\overline{\mathbf{1 0}}_{H}\left[M^{\prime} 10_{1}+\lambda^{\prime} \mathbf{2 4} 10_{2}\right]+h 10_{1} \overline{\mathbf{H}} \overline{\mathbf{5}}_{1} .
$$

Now the soft masses of the squarks and sleptons embedded in the 10s will split as in eq. (7) depending on their hypercharge. As shown in Fig. 2 for $\Delta m^{2} / m^{2} \equiv\left(m_{\tilde{t}_{R}}^{2}-m_{\tilde{t}_{L}}^{2}\right) / m_{\tilde{t}_{R}}^{2}$, these splittings can be much larger than for the fiveplet since the differences in hypercharges are larger in the decouplet.

In the fermion sector eq. (11) will be modified to

$$
\frac{m_{b}}{m_{\tau}}=\frac{s_{b_{L}}}{s_{\tau_{R}}}=\frac{1}{6} \sqrt{\frac{1+36 \rho^{\prime 2}}{1+\rho^{\prime 2}}}
$$

where $\rho^{\prime}=\lambda^{\prime} V_{24} / M^{\prime}$. Now the ratio of $m_{b} / m_{\tau}$ can range from $1 / 6$ to 1 and gives even more flexibility to this prediction. This idea can also be usefully applied to the second generation ratio $m_{s} / m_{\mu}$ and avoid the potentially problematic GUT relation $m_{s}=m_{\mu}$.

It is easy to see that if both the fiveplets and the decouplets are linear combinations of representations, i.e., the superpotential is the sum of eqs. (3) and (12), then we get the fermion mass ratio

$$
\frac{m_{b}}{m_{\tau}}=\frac{s_{b_{R}} s_{b_{L}}}{s_{\tau_{R}} s_{\tau_{L}}}=\frac{1}{9} \sqrt{\frac{\left(1+9 \rho^{2}\right)\left(1+36 \rho^{\prime 2}\right)}{\left(1+4 \rho^{2}\right)\left(1+\rho^{\prime 2}\right)}} .
$$

This ranges from 1/9 to 1 and can give even larger particle mass-splittings.

\section{A SO(10) model with $1<\mathrm{h}_{\mathrm{t}} / \mathrm{h}_{\mathrm{b}}<3$}

The previous examples can be easily adapted to $\mathrm{SO}(10)$. The Higgs sector consists of only two multiplets, $\mathbf{4 5} \ni \mathbf{2 4}$ and $\mathbf{1 0} \ni\{\mathbf{H}, \overline{\mathbf{H}}\}$, and the matter fields are embedded in the $16 \mathrm{~s}$ spinor representations. Since the $\mathbf{4 5}$ is in the adjoint 
representation of $\mathrm{SO}(10)$, its $\mathrm{VEV}$ can point in any direction of the two dimensional subspace of $\mathrm{SO}(10)$ generators that commutes with $\mathrm{SU}(3) \times \mathrm{SU}(2) \times \mathrm{U}(1)$. One possible direction, as in the previous example, is the hypercharge direction $\mathbf{Y}$. Here we will consider the case where the VEV of the $\mathbf{4 5}$ points in the $\mathbf{X}$-direction, where $\mathbf{X}$ is the $\mathrm{SO}(10)$ generator that commutes with the $\mathrm{SU}(5)$ subgroup, i.e. $\langle\mathbf{4 5}\rangle=V_{45} \mathbf{X}$. The superpotential in now given by

$$
W=\overline{\mathbf{1 6}}_{H}\left[M 16_{1}+\lambda 4516_{2}\right]+h 16_{2} 1016_{2} .
$$

Notice we have now coupled the $\mathbf{1 6}_{2}$ instead of the $\mathbf{1 6}_{1}$ to the Higgs $\mathbf{1 0}$. As in the previous model, the light quarks and leptons arise from the linear combination, $-\sin \theta_{X} \mathbf{1 6}_{1}+\cos \theta_{X} \mathbf{1 6}_{2}$, where now the mixing angle is

$$
\sin \theta_{X}=\frac{\rho \mathbf{X}}{\sqrt{1+\rho^{2} \mathbf{X}^{2}}}
$$

where $\rho=\lambda V_{45} / M$. The $X$-charges of the quarks and leptons are

$$
X_{a}=(1,1,-3,-3,1,5) \text { for } a=\left(Q, U^{c}, D^{c}, L, E^{c}, \nu^{c}\right) \text {, }
$$

where $Q$ is the quark $\mathrm{SU}(2)$-doublet and $U^{c}, E^{c}$ and $\nu^{c}$ are the quark and lepton singlets. The scalar masses are split according to

$$
m_{\tilde{a}}^{2}=m_{2}^{2}+s_{a}^{2}\left(m_{1}^{2}-m_{2}^{2}\right)
$$

where $s_{a} \equiv \sin \theta_{X_{a}}$ and $m_{i}^{2}$ is the soft mass of $\mathbf{1 6}_{i}$. These scalar mass-splittings preserve $\mathrm{SU}(5)$ since the $\langle\mathbf{4 5}\rangle$ does not break this subgroup of $\mathrm{SO}(10)$.

The fermion masses in this model are proportional to $c_{a}=\sqrt{1-s_{a}^{2}}$ since we coupled the Higgs $\mathbf{1 0}$ to the $\mathbf{1 6}_{2}$. For the third family, we have

$$
\frac{h_{t}}{h_{b}}=\frac{c_{t_{R}} c_{t_{L}}}{c_{b_{R}} c_{b_{L}}}=\sqrt{\frac{1+9 \rho^{2}}{1+\rho^{2}}},
$$

which has a maximum value of 3 for $\rho \rightarrow \infty$. Nevertheless, we cannot take very large values of $\rho$ since the top mass is given by

$$
m_{t}=\frac{h\langle\mathbf{1 0}\rangle}{1+\rho^{2}}
$$

\footnotetext{
${ }^{6}$ Since this direction preserves $\mathrm{SU}(5)$, we still have bottom-tau unification.
} 
and decreases when increasing $\rho$. To avoid a light top or a too large $h$-Yukawa coupling, the value of $\rho$ cannot be larger than $1-2$ that leads to $h_{t} / h_{b} \sim 2.2-2.7$.

Therefore this minimal extension of the simplest $\mathrm{SO}(10)$ theory can lead to a partial explanation of the large top-bottom mass ratio, allows for moderate values of $\tan \beta \sim 20$ and (since $h_{t}>h_{b}$ ) accommodates radiative electroweak breaking without an extreme fine-tuning. In contrast the minimal $\mathrm{SO}(10)$ theory has $h_{t}=h_{b}=h_{\tau}=h_{\nu}$; this implies that $\tan \beta$ is large, $\tan \beta \sim 40-60$, and a severe fine-tuning is required to get the correct electroweak symmetry breaking [15]. Another difference with the minimal $\mathrm{SO}(10)$ theory has to do with neutrinos. In our theory, from eq. (17), one has that the Dirac-type Yukawa coupling of the neutrino is very suppressed

$$
\frac{h_{\nu}}{h_{t}}=\frac{c_{\nu_{R}} c_{\nu_{L}}}{c_{t_{R}} c_{t_{L}}}=\frac{1+\rho^{2}}{\sqrt{\left(1+25 \rho^{2}\right)\left(1+9 \rho^{2}\right)}},
$$

and therefore does not modify the value of $m_{b} / m_{\tau}$ even if the right-handed neutrino mass is light $\ll 10^{16} \mathrm{GeV}$ and $\nu_{L}$ is cosmologically interesting. In contrast in $\mathrm{SO}(10)$ with $h_{t}=h_{\nu}$, the neutrino coupling affects $m_{b} / m_{\tau}$; this

effectively excludes neutrinos from being cosmologically relevant for values of $\tan \beta<10$ [12]. Finally, to accommodate $m_{b} / m_{\tau}$ our theory disfavors large values for the strong coupling and small values for the bottom mass -unless there are significant low-energy contributions to $m_{b}$ [15, 16].

\section{Horizontal splittings with horizontal symme- tries}

The mechanism of this paper can also generate horizontal mass-splittings in theories with horizontal symmetries. As an example, consider a $\mathrm{SO}(10) \times \mathrm{SU}(3)_{H}$ theory where the $\mathrm{SU}(3)_{H}$ is a horizontal symmetry under which the three families transform as a triplet. The superpotential is given by

$$
\begin{aligned}
W & =(\overline{\mathbf{1 6}}, \overline{\mathbf{3}})_{H}\left[M(\mathbf{1 6}, \mathbf{3})_{1}+\lambda(\mathbf{4 5}, \mathbf{8})(\mathbf{1 6}, \mathbf{3})_{2}\right] \\
& +h(\mathbf{1 6}, \mathbf{3})_{2}(\mathbf{1 0}, \overline{\mathbf{6}})(\mathbf{1 6}, \mathbf{3})_{2} .
\end{aligned}
$$

Depending on the direction of the VEV of the $(\mathbf{4 5}, \mathbf{8})$, we have different possibilities of splitting the masses of the three families. Constraints from flavor 
violating processes requires near degeneracy between the first and second family scalars [1]; this can be guaranteed if the VEV of the $(45,8)$ points in the direction $T_{8}=\operatorname{diag}(1,1,-2)$ of $\mathrm{SU}(3)_{H}$ which preserves $\mathrm{SU}(2)_{H}$. If respect $\mathrm{SO}(10)$, the VEV of $(\mathbf{4 5}, \mathbf{8})$ points in the $\mathrm{X}$-direction [7, the sparticle masses are given by eq. (18) where now the mixing angle is given by

$$
s_{a(i)}=\frac{\rho X_{a}\left[T_{8}\right]_{i}}{\sqrt{1+\rho^{2} X_{a}^{2}\left[T_{8}\right]_{i}^{2}}},
$$

where $X_{a}$ is given in eq. (17) and $\left[T_{8}\right]_{i}=(1,1,-2)$ for $i=\left(1^{\text {st }}, 2^{\text {nd }}, 3^{\text {rd }}\right.$ family $)$. The sparticles of the third family are split from those of the first and second families because the $\mathrm{SU}(3)_{H}$ symmetry is broken.

\section{Summary}

We presented a mechanism that leads to interesting splittings of particle and sparticle masses at the same time. It implies that sparticles belonging to the same generation can have significant splittings at the unification scale. How general is the mechanism we presented? It occurs in a large class of theories which, near the unification mass, have $N+3$ left-handed and $N$ right-handed families which subsequently combine to leave 3 light families. Because the physics that determines the light states that we call quarks and leptons breaks the unified group, the tree level particle and sparticle masses in general do not obey any GUT relations 1 and lead to splittings. Therefore the sparticle spectroscopy that results is richer and may provide us with more detailed information about the physics near the unification mass than in minimal theories with universality.

\section{Acknowledgements}

We thank M. Carena and C. Wagner for discussions on $b-\tau$ unification.

\footnotetext{
${ }^{7}$ The fermion mass relations in this model will be like those of the previous section. Since these relations work best for the third family, it is simplest to assume that only $\left(\mathbf{1 0}, \overline{\mathbf{6}}_{33}\right)$ is light.

${ }^{8}$ If they do, it is an indication of some unexpected simplicity that should be understood. The absence of large flavor violations is an indication of partial simplicity in the horizontal direction for some of the sparticles of the two lightest generations.
} 


\section{References}

[1] S. Dimopoulos and H. Georgi, "Supersymmetric GUTs", p. 285, Second Workshop on Grand Unification, University of Michigan, Ann Arbor, April 24-26 (1981), eds. J. Leveille, L. Sulak and D. Unger, Birkhauser (1981); S. Dimopoulos and H. Georgi, Nucl. Phys. B193 (1981) 150.

[2] S. Dimopoulos, S. Raby and F. Wilczek, Phys. Rev. D24 (1981) 1681.

[3] N. Sakai, Z. Phys. C11 (1981) 153; L. Ibañez and G.G. Ross, Phys. Lett. B105 (1981) 439; M.B. Einhorn and D.R.T. Jones, Nucl. Phys. B196 (1982) 475; W.J. Marciano and G. Senjanovic, Phys. Rev. D25 (1982) 3092 .

[4] Excellent recent analyses are P. Langacker and N. Polonsky, Phys. Rev. D47 (1993) 4028; L.J. Hall and U. Sarid, Phys. Rev. Lett. 70 (1993) 2673.

[5] L.J. Hall, V.A. Kostelecky and S. Raby, Nucl. Phys. B267 (1986) 415;

H. Georgi, Phys. Lett. B169 (1986) 231.

[6] N. Polonsky and A. Pomarol, Phys. Rev. Lett. 73 (1994) 2292; Preprint hep-ph/9410231.

[7] L. Ibañez and D. Lust, Nucl. Phys. B382 (1992) 305; V. Kaplunovsky and J. Louis, Phys. Lett. B306 (1993) 269; A. Brignole, L. Ibañez and C. Muñoz, Nucl. Phys. B422 (1994) 125; S. Ferrara, C. Kounnas and F. Zwirner, CERN Preprint TH-7192-94; J. Louis and Y. Nir, München Preprint LMUTPW 94-17.

[8] M. Drees, Phys. Lett. B181 (1986) 279; J. S. Hagelin and S. Kelley, Nucl. Phys. B342 (1990) 95; Y. Kawamura, H. Murayama and M. Yamaguchi, Phys. Lett. B324 (1994) 52; H.C. Cheng and L.J. Hall, Berkeley Preprint LBL-35950.

[9] M.S. Chanowitz, J. Ellis and M.K. Gaillard, Nucl. Phys. B128 (1977) 506.

[10] See for example, P. Langacker and N. Polonsky, Phys. Rev. D49 (1994) 1454. 
[11] See for example, V. Barger, M.S. Berger and P. Ohmann, Phys. Rev. D49 (1994) 4908; M. Carena, M. Olechowski, S. Pokorski and C.E.M. Wagner, Nucl. Phys. B419 (1994) 213; and Ref. [6].

[12] F. Vissani and A.Y. Smirnov, SISSA Preprint SISSA-63/94/EP; A. Brignole, H. Murayama and R. Rattazzi, Berkeley Preprint LBL-35774.

[13] S. Dimopoulos, Phys. Lett. B129 (1983) 417.

[14] G. Anderson, S. Dimopoulos, L.J. Hall, S. Raby and G.D. Starkman, Phys. Rev. D49 (1994) 3660.

[15] L.J. Hall, R. Rattazzi and U. Sarid, Phys. Rev. D50 (1994) 7048; Preprint hep-ph/9405313.

[16] M. Carena, M. Olechowski, S. Pokorski and C.E.M. Wagner, Nucl. Phys. B426 (1994) 269.

\section{Figure Captions}

Fig. 1: Scalar mass-splitting $\Delta m^{2} / m^{2} \equiv\left(m_{\tilde{\tau}_{L}}^{2}-m_{\tilde{b}_{R}}^{2}\right) / m_{\tilde{\tau}_{L}}^{2}$ as a function of $\rho=\lambda V_{24} / M$ and for different values of $\Delta=m_{2}^{2} /\left(m_{1}^{2}-m_{2}^{2}\right)$. The dashed line corresponds to the the ratio $m_{b} / m_{\tau}$.

Fig. 2: Scalar mass-splitting $\Delta m^{2} / m^{2} \equiv\left(m_{\tilde{t}_{R}}^{2}-m_{\tilde{t}_{L}}^{2}\right) / m_{\tilde{t}_{R}}^{2}$ as a function of $\rho^{\prime}=\lambda^{\prime} V_{24} / M^{\prime}$ and for different values of $\Delta=m_{2}^{2} /\left(m_{1}^{2}-m_{2}^{2}\right)$. The dashed line corresponds to the the ratio $m_{b} / m_{\tau}$. 
Fig. 1

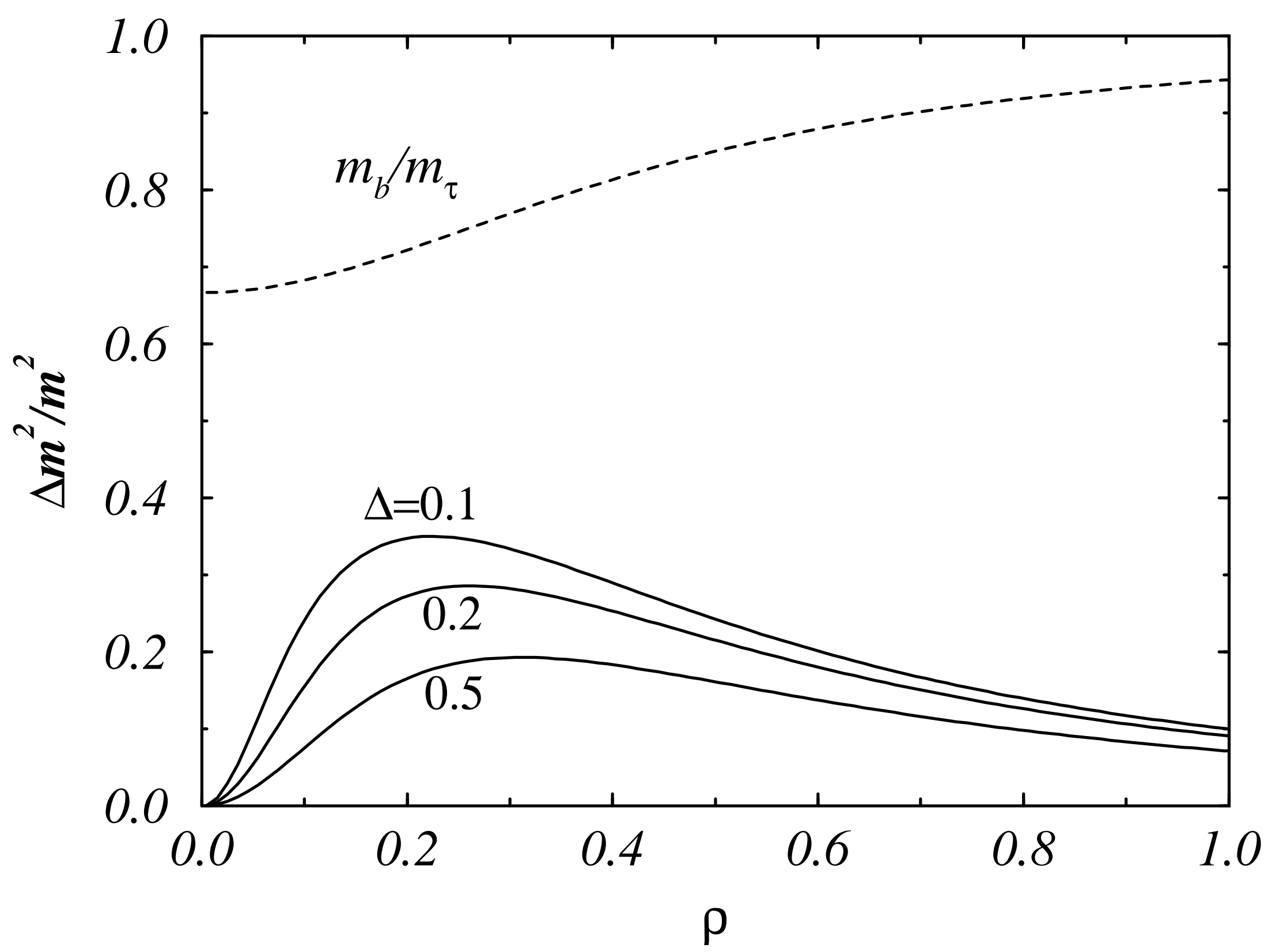


Fig. 2

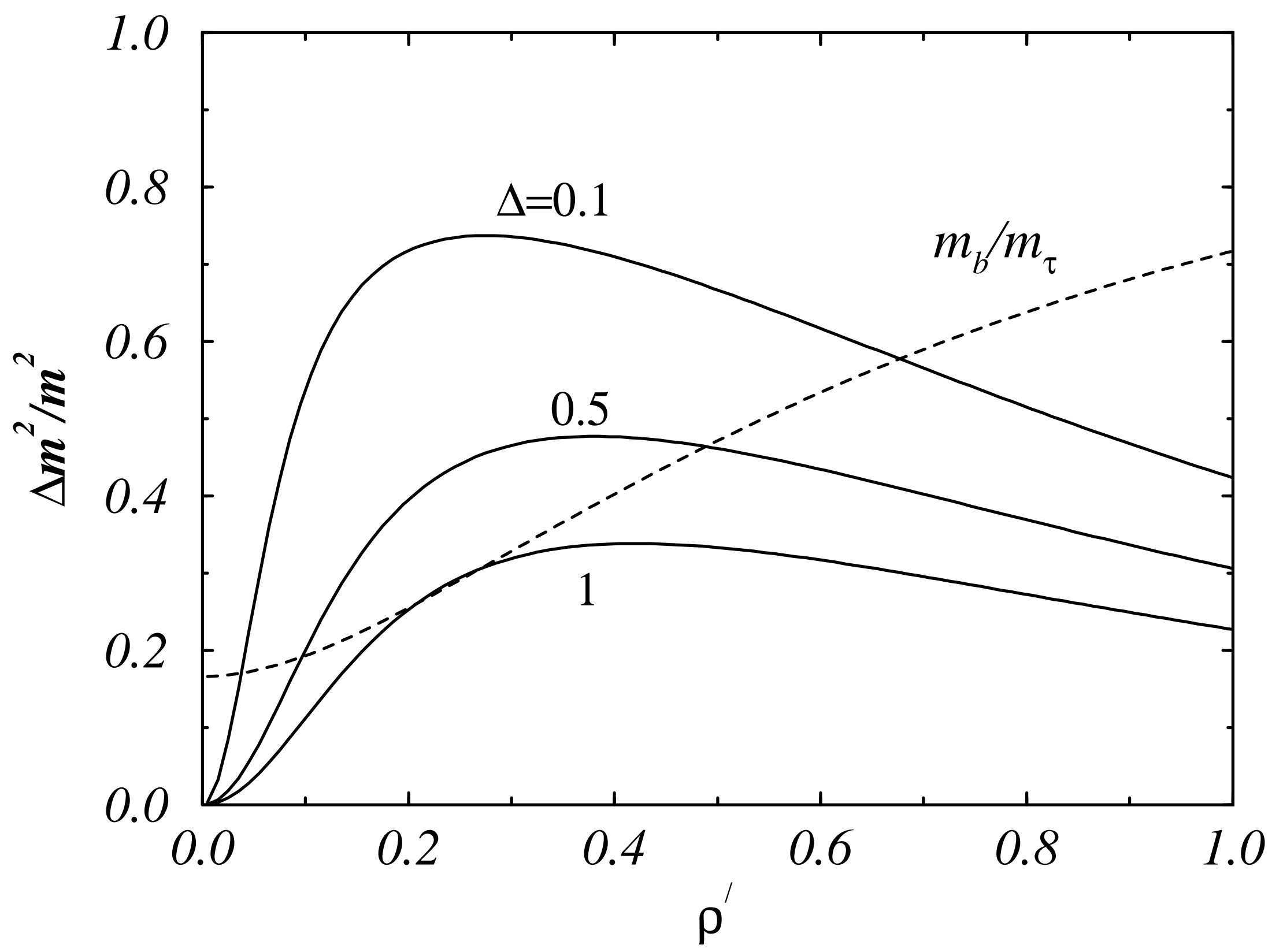

